\title{
Interação genótipo $x$ ambiente e estimativas de parâmetros genéticos dos pesos aos 205 e 365 dias de idade de bovinos Nelore
}

\author{
[Genotype by environment interaction and genetic parameter estimates for 205 and 365 \\ body weight of Nelore cattle]

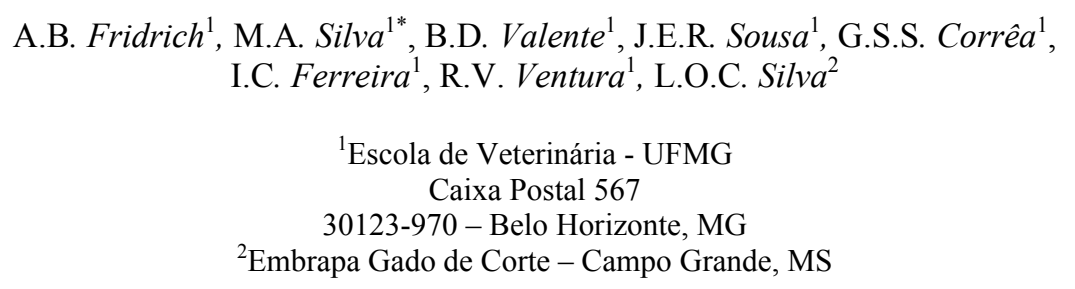

\section{RESUMO}

Dados de pesos aos 205 (P205) e 365 (P365) dias de idade, de 46.408 animais Nelore, nascidos entre 1976 e 2000, provenientes de 530 rebanhos dos diversos estados brasileiros, foram utilizados para avaliar as interações genótipo x ambiente (IGA) e estimar herdabilidades direta e materna dos pesos pelo método da máxima verossimilhança restrita. $O$ modelo estatístico utilizado incluiu efeitos fixos de grupo contemporâneo, idade da vaca ao parto (covariável) e efeitos aleatórios genéticos aditivo direto e materno. As correlações genéticas, estimadas para as características P205 e P365, cada uma considerada como característica distinta em cada uma das regiões Sul (S), Sudeste (SE), Centro-Oeste (CO), Norte (N) e Nordeste (NE), foram, respectivamente, 0,86 e $0,84,0,64$ e $0,35,0,75$ e $0,42,0,79$ e $0,86,0,92$ e 0,81 , 0,95 e $0,83,0,83$ e $1,00,0,88$ e $0,81,0,33$ e $0,85,0,63$ e 0,99 para $\mathrm{S}$ e $\mathrm{SE}, \mathrm{S}$ e $\mathrm{CO}, \mathrm{S}$ e $\mathrm{N}, \mathrm{S}$ e NE, SE e $\mathrm{CO}, \mathrm{SE}$ e N, SE e NE, CO e N, CO e NE e N e NE. As baixas correlações genéticas indicaram que há efeito da interação genótipo $x$ ambiente nas combinações que envolvem as regiões $\mathrm{S} / \mathrm{CO}, \mathrm{S} / \mathrm{N}, \mathrm{S} / \mathrm{NE}$, $\mathrm{CO} / \mathrm{NE}$ e N/NE para P205 dias de idade e S/CO e S/N para P365 dias de idade, havendo, portanto, necessidade de avaliação genética regional quando se consideram regiões bastante distintas.

Palavras-chave: bovino, Nelore, herdabilidade, interação genótipo x ambiente

\begin{abstract}
Body weight records at $205(205 \mathrm{BW})$ and $365(365 \mathrm{BW})$ days of age of 46,408 Nelore animals, born from 1976 to 2000 period in 530 Nelore herds of several states of Brazil, were used to evaluate genotype by environment interactions and to estimate genetic and maternal heritability by restricted maximum likelihood methodology. The statistical model included the fixed effects of contemporary group and age of cow (covariate), and the random direct and maternal additive genetic effects. The genetic correlation for $205 \mathrm{BW}$ and 365BW, each one considered as different traits in each of the South (S), Southeast (SE), Central west (CO), North $(N)$ and Northeast $(N E)$ regions were 0.86 and $0.84,0.64$ and $0.35,0.75$ and $0.42,0.79$ and $0.86,0.92$ and $0.81,0.95$ and $0.83,0.83$ and $1.00,0.88$ and $0.81,0.33$ and $0.85,0.63$ and 0.99 for $S / S E, S / C O, S / N, S / N E, S E / C O, S E / N, S E / N E, C O / N, C O / N E$ and N/NE, respectively. There is a significant genotype by environment interaction for 205BW in the combination involving the $S / C O, S / N$, $S / N E$, CO/NE, and N/NE regions. There are significant genotype by environment interaction effects in the combination involving the $S / C O$ and $S / N$ regions for $365 \mathrm{BW}$. Based on genetic x environment interaction results, regional genetic evaluation is recommend for the very distinct regions.
\end{abstract}

Keywords: bovine, Nelore, heritability, genotype by environment interaction

Recebido em 5 de outubro de 2007

Aceito em 3 de junho de 2008

*Autor para correspondência (corresponding author)

E-mail: martinho@vet.ufmg.br 


\section{INTRODUÇÃO}

A investigação da existência de interação genótipo x ambiente (IGA) é essencial para os programas de melhoramento genético dos animais. Os estudos deste tipo de interação começaram a partir do século XX e estão basicamente relacionados ao ambiente no qual a seleção deve ser praticada, se no ambiente em que os animais serão criados ou no ambiente melhorado que permitiria que os animais expressassem todo o seu potencial genético.

Hammond (1947) propunha que os animais deveriam ser selecionados em ambiente que permitisse sua máxima expressão, mas Falconer (1952) sugeria que o conjunto de genes responsáveis pela expressão de determinada característica poderia variar em diferentes ambientes de criação, caso esta característica apresentasse IGA.

O Brasil, entre os países que apresentam grande extensão territorial, o clima, o manejo e a alimentação dos animais podem variar muito entre as regiões. $\mathrm{O}$ valor genético de diferentes animais pode apresentar diferentes sensibilidades a esta variação ambiental, o que caracteriza a existência de IGA. Dessa maneira, diferentes regiões podem apresentar diferentes ordens de classificação dos valores genéticos preditos dos animais. Isso faz com que avaliações genéticas com base em modelos que consideram características mensuradas em diferentes ambientes como uma só característica, como os modelos utilizados por Marques et al. (2000) e Pereira et al. (2001), possam apresentar menor eficiência na predição de valores genéticos para determinados ambientes.

Os resultados obtidos por Alencar et al. (2005), por meio de componentes principais, indicam interação touro $\mathrm{x}$ época de nascimento significativa para pesos e ganhos de pesos de animais Canchim até os 12 meses de idade, e sugerem que essa interação deva ser considerada nos estudos de avaliação genética.

Fridrich et al. (2005), ao estudarem pesos aos 205 e 365 dias de idade de animais Tabapuã nas diversas regiões brasileiras e considerarem a expressão da mesma característica em ambientes diferentes como sendo expressão de diferentes características, obtiveram resultados que indicam existência de IGA, principalmente para as combinações de regiões que envolvem a região nordeste.

Ribeiro (2006) avaliou o peso aos 205 e 550 dias de idade, além do ganho de peso entre 205 e 550 dias de idade de animais Nelore de diferentes rebanhos do estado de São Paulo e um rebanho do estado do Mato Grosso do Sul. Ao considerar, informações provenientes de um universo de ambientes de criação mais estreito, o autor estimou correlações genéticas entre observações da mesma característica coletadas em ambientes diferentes que variaram entre 0,82 a 1,00 . Esses resultados sugerem pequeno ou nenhum efeito da IGA nos rebanhos estudados. Ferreira et al. (2001) estimaram altas correlações genéticas para peso aos 120 dias e para peso aos 205 dias de idade em animais Nelore criados em duas fazendas localizadas em regiões bem distintas do estado de Minas Gerais. As correlações obtidas foram, respectivamente, 0,96 e 0,95 , estimativas que sugerem ausência de efeito importante de IGA. Por outro lado, para o peso aos 550 dias, a correlação estimada foi 0,53 , indicando efeito de IGA.

Portanto, o assunto é ainda controverso e merece estudos mais detalhados que considerem maior amplitude de ambientes e envolvam maior número de rebanhos distribuídos nos vários estados brasileiros, para melhor avaliação genética dos animais, o que constitui a base de qualquer programa de melhoramento.

Neste trabalho, investigou-se a existência de interação genótipo $\mathrm{x}$ ambiente nos pesos aos 205 e 365 dias de idade de bovinos Nelore, considerando peso (205 ou 365 dias de idade) como característica distinta em cada região, bem como estimaram-se os parâmetros genéticos desses pesos.

\section{MATERIAL E MÉTODOS}

Os dados utilizados neste estudo são de 46.408 animais Nelore, nascidos entre 1976 e 2000, provenientes de 530 rebanhos dos diversos estados brasileiros incluídos no Controle de Desenvolvimento Ponderal da Associação Brasileira de Criadores de Zebu (ABCZ) e cedidos pela Embrapa - Gado de Corte, localizada em Campo Grande, MS. 
As características estudadas foram os pesos aos 205 (P205) e aos 365 dias de idade (P365), sendo os pesos padronizados por interpolação. Foram formados três arquivos para as análises. O primeiro arquivo continha a identificação do animal, do pai e da mãe; o segundo e o terceiro arquivo, continham a identificação de animal e mãe, grupo contemporâneo, idade da mãe ao parto (covariável) e os pesos aos 205 e 365 dias de idade, respectivamente, considerados como características distintas em cada região.

Para avaliar o efeito da IGA sobre as características analisadas nas diversas regiões brasileiras, os arquivos foram constituídos apenas com informações de animais criados exclusivamente em pasto. Os estados foram agrupados em cinco regiões distintas: 1-Sul (S),
2-Sudeste (SE), 3-Centro-Oeste (CO), 4-Norte (N) e 5-Nordeste (NE). Foram consideradas duas estações de nascimento.

Foram também definidos os "touros de conexão", isto é, touros com número mínimo de três progênies em cada uma de duas ou mais regiões. Os grupos contemporâneos (GC) foram formados por ano de nascimento, propriedade, estação de nascimento e sexo, totalizando 4.490 grupos contemporâneos.

As análises estatísticas dos pesos foram realizadas com o auxílio do procedimento general linear models (GLM), contido no SAS (Statistical..., 1997). Na Tab. 1, encontram-se as estatísticas descritivas das características da amostra estudada nas regiões.

Tabela 1. Estatística descritiva dos arquivos com informações de pesos aos 205 e 365 dias de idade de animais Nelore nas diversas regiões

\begin{tabular}{lccccc}
\hline & Sul & Sudeste & Centro-Oeste & Norte & Nordeste \\
\hline $\mathrm{N}^{\circ}$ animais & 642 & 26.716 & 13.738 & 3.746 & 1.566 \\
$\mathrm{~N}^{\circ}$ animais na A & 59.507 & 59.507 & 59.507 & 59.507 & 59.507 \\
$\mathrm{Média}^{-1} \mathrm{DP}$ P205 & $168,46 \pm 23,8$ & $167,76 \pm 28,1$ & $167,11 \pm 26,8$ & $167,20 \pm 24,6$ & $173,75 \pm 28,9$ \\
Média \pm DP P365 & $211,77 \pm 33,3$ & $219,85 \pm 36,7$ & $217,18 \pm 36,6$ & $215,25 \pm 34,7$ & $227,88 \pm 39,9$ \\
$\mathrm{CV} \%$ - P205 & 14,15 & 16,75 & 16,10 & 14,70 & 16,66 \\
$\mathrm{CV} \%$ - P365 & 15,70 & 16,69 & 16,86 & 16,10 & 17,54 \\
\hline
\end{tabular}

$\mathrm{A}^{-1}=$ matriz de numeradores dos coeficientes de parentesco é igual para todos os arquivos.

$\mathrm{DP}=$ desvio-padrão; $\mathrm{CV}=$ coeficiente de variação.

Para estimação dos componentes de (co)variância e predição dos valores genéticos, utilizou-se o método de máxima verossimilhança restrita aplicado a um modelo animal, usando o programa Multiple Trait Derivative-Free Restricted Maximum Likelihood (MTDFREML), desenvolvido por Boldman et al. (1995). Para estimação dos parâmetros genéticos, a saber, componentes de variância genéticos aditivos, maternos, covariância e correlação entre efeitos diretos e maternos, foram feitas análises unicaracterísticas com o emprego do modelo que incluiu o efeito fixo de GC, a idade da vaca ao parto como covariável (efeito linear e quadrático) e os efeitos genéticos aditivos direto e materno, bem como a covariância entre efeitos genético aditivo e o materno .

Os efeitos da IGA foram obtidos pelas correlações genéticas entre cada peso, considerando a mesma característica (P205 ou P365) como característica distinta nas várias regiões. Os dados foram analisados considerando-se duas características (análise bicaracterística) em cada análise, segundo o modelo:

$\mathrm{y}=\mathrm{Xb}+\mathrm{Zu}+\mathrm{Wm}+\mathrm{e}$;

em que:

$\mathrm{y}=$ vetor de pesos padronizados para 205 ou 365 dias de idade dos animais.

$\mathrm{X}, \mathrm{Z}$ e $\mathrm{W}=$ matrizes de incidência dos efeitos fixos e aleatórios;

$\mathrm{b}=$ vetor de efeito fixo de grupo contemporâneo (GC) e da idade da vaca ao parto como covariável; $\mathrm{u}=$ vetor dos efeitos aleatórios de valores genéticos aditivos diretos do animal;

$\mathrm{m}=$ vetor dos efeitos aleatórios de valores genéticos aditivos maternos e;

$\mathrm{e}=$ vetor dos erros aleatórios associados às observações.

Outra estatística utilizada para o estudo da interação genótipo $\mathrm{x}$ ambiente foi a correlação de posto (Spearman) dos valores genéticos preditos para cada par de combinações das regiões estudadas, para P205 e P365. 


\section{RESULTADOS E DISCUSSÃO}

As variâncias, covariâncias, herdabilidades $\left(h^{2}\right)$ e correlação genética entre os efeitos genéticos aditivos direto e materno $\left(\mathrm{r}_{\mathrm{am}}\right)$ dos pesos aos 205 (P205) e aos 365 (P365) dias de idade, estimadas para cada região, são apresentadas, respectivamente, nas Tab. 2 e 3.

As estimativas de parâmetros genéticos variaram muito em todas as regiões para ambas as características. Esta grande variação pode ter ocorrido em razão do número diferente de informações em cada região. $\mathrm{Na}$ região $\mathrm{CO}$, a segunda com maior número de animais, foram observados valores mais elevados da $\sigma_{a}^{2}$ e $\sigma_{m}^{2}$ para ambas as características P205 (125,92 e
116,69) e P365 (189,76 e 111,75). Grande heterogeneidade de variância genética aditiva direta e materna foi observada para P205 e P365 entre as regiões $\mathrm{SE}$ e $\mathrm{CO}$, as regiões com maior número de animais. Esta heterogeneidade não foi observada por Valente et al. (2008), que estimaram estruturas de covariância de peso em função da idade de bovinos Nelore das regiões $\mathrm{SE}$ e CO. Na região $\mathrm{S}$ foi observada menor $\sigma^{2}$, tanto para P205 $(2,69)$ quanto para P365 $(26,94)$. As análises na região $\mathrm{S}$ forneceram valores atípicos em relação às outras regiões para os parâmetros genéticos dos animais, mas essa foi a região que apresentou o menor número de dados, automaticamente, reflexo dos menores números de rebanhos, touros e de matrizes.

Tabela 2. Variâncias genéticas aditivas diretas $\left(\sigma_{\mathrm{a}}^{2}\right)$, genéticas aditivas maternas $\left(\sigma_{\mathrm{m}}^{2}\right)$, fenotípicas $\left(\sigma_{\mathrm{p}}^{2}\right)$, residuais $\left(\sigma_{\mathrm{e}}^{2}\right)$, covariância entre os efeitos genéticos aditivos direto e materno $\left(\sigma_{\mathrm{am}}\right)$, herdabilidade direta $\left(\mathrm{h}_{\mathrm{a}}^{2}\right)$ e materna $\left(\mathrm{h}_{\mathrm{m}}^{2}\right)$ e correlação entre os efeitos genéticos aditivos direto e materno $\left(\mathrm{r}_{\mathrm{am}}\right)$ obtidas em análises unicaracterísticas do peso aos 205 (P205) dias de idade, em cada uma das regiões Sul (S), Sudeste (SE), Centro-Oeste (CO), Norte (N) e Nordeste (NE)

\begin{tabular}{lllllllll} 
Característica & $\sigma_{\mathrm{a}}^{2}$ & $\sigma_{\mathrm{m}}^{2}$ & $\sigma_{\mathrm{p}}^{2}$ & $\sigma_{\mathrm{e}}^{2}$ & $\sigma_{\mathrm{am}}$ & $\mathrm{h}_{\mathrm{a}}^{2}$ & $\mathrm{~h}_{\mathrm{m}}{ }^{2}$ & $\mathrm{r}_{\mathrm{am}}$ \\
\hline P205 (S) & 2,69 & 69,52 & 388,33 & 329,80 & $-13,69$ & 0,01 & 0,18 & $-1,00$ \\
P205 (SE) & 74,13 & 77,53 & 465,71 & 334,85 & $-20,81$ & 0,16 & 0,17 & $-0,27$ \\
P205 (CO) & 125,92 & 116,69 & 437,59 & 254,58 & $-59,61$ & 0,29 & 0,27 & $-0,49$ \\
P205 (N) & 83,76 & 115,90 & 394,84 & 233,57 & $-38,39$ & 0,21 & 0,29 & $-0,39$ \\
P205 (NE) & 57,20 & 31,41 & 355,59 & 283,11 & $-15,96$ & 0,16 & 0,09 & $-0,38$ \\
\hline
\end{tabular}

Tabela 3. Variâncias genéticas aditivas diretas $\left(\sigma_{\mathrm{a}}^{2}\right)$, genéticas aditivas maternas $\left(\sigma_{\mathrm{m}}^{2}\right)$, fenotípicas $\left(\sigma_{\mathrm{p}}^{2}\right)$, residuais $\left(\sigma_{\mathrm{e}}^{2}\right)$, covariância entre os efeitos genéticos aditivos direto e materno $\left(\sigma_{\mathrm{am}}\right)$, herdabilidade direta $\left(\mathrm{h}_{\mathrm{a}}^{2}\right)$ e materna $\left(\mathrm{h}_{\mathrm{m}}^{2}\right)$ e correlação entre os efeitos direto e materno $\left(\mathrm{r}_{\mathrm{am}}\right)$ obtidas em análises unicaracterísticas do peso aos 365 (P365) dias de idade, em cada uma das regiões Sul (S), Sudeste (SE), Centro-Oeste (CO) Norte (N) e Nordeste (NE)

\begin{tabular}{lllllllll} 
Característica & $\sigma_{\mathrm{a}}^{2}$ & $\sigma_{\mathrm{m}}^{2}$ & $\sigma_{\mathrm{p}}^{2}$ & $\sigma_{\mathrm{e}}^{2}$ & $\sigma_{\mathrm{am}}$ & $\mathrm{h}_{\mathrm{a}}^{2}$ & $\mathrm{~h}_{\mathrm{m}}^{2}$ & $\mathrm{r}_{\mathrm{am}}$ \\
\hline P365 (S) & 26,94 & 94,44 & 537,59 & 416,17 & 0,04 & 0,05 & 0,18 & 0,00 \\
P365 (SE) & 91,39 & 62,36 & 705,65 & 544,49 & 7,41 & 0,13 & 0.09 & 0.10 \\
P365 (CO) & 189,76 & 111,75 & 652,63 & 414,98 & $-63,85$ & 0,29 & 0,17 & $-0,44$ \\
P365 (N) & 136,82 & 46,35 & 678,79 & 480,26 & 15,36 & 0,20 & 0,07 & 0,19 \\
P365 (NE) & 32,94 & 55,68 & 569,04 & 503,52 & $-23,10$ & 0,06 & 0,10 & $-0,54$ \\
\hline
\end{tabular}

Valores positivos de correlação entre efeitos genético aditivo direto e materno foram estimados somente para P365 nas regiões SE e $\mathrm{N}$, o que indica que ao se utilizar, nessas regiões, o valor genético aditivo direto no estabelecimento do critério de seleção não haverá impacto negativo no mérito genético aditivo materno dos animais. Os valores estimados da correlação na região S, referente ao P205 e P365, devem ser encarados com reserva, em razão do baixo número de dados existentes para essa região. As $r_{a m}$ foram negativas para P205 em todas as regiões e para $\mathrm{P} 365$ nas regiões $\mathrm{CO}$ e $\mathrm{NE}$, o que evidencia um antagonismo entre os efeitos genéticos direto e materno, ou seja, ao se utilizar, como critério de seleção, o valor genético aditivo direto, resultará em impacto negativo no mérito genético aditivo materno dos animais

Correlações entre efeito genético aditivo direto e materno negativas para P205 (-0,70) e P365 (- 
$0,74)$ foram encontradas por Ribeiro et al. (2001), ao trabalharem com bovinos Nelore no estado da Paraíba. Na região $\mathrm{S}$, a $\mathrm{r}_{\mathrm{am}}$ para $\mathrm{P} 365$ foi igual a zero, o que indica dissociação entre os efeitos genéticos aditivos direto e materno. Fridrich et al. (2005) encontraram $r_{a m}$ iguais a zero nas regiões $\mathrm{CO}$ e NE para a característica P205, e na região S para P365, ao trabalharem com bovinos Tabapuã.

De modo geral, as estimativas de herdabilidade direta $\left(\mathrm{h}_{\mathrm{a}}^{2}\right)$ foram menores do que as herdabilidades maternas $\left(\mathrm{h}_{\mathrm{m}}^{2}\right)$ para $\mathrm{P} 205$, e o inverso ocorreu para P365, o que indica grande influência do efeito materno sobre P205, uma vez que existe maior dependência do bezerro em relação às suas mães até a desmama.

As estimativas de $\mathrm{h}^{2}$ a para P205 foram semelhantes às encontradas por Souza et al. (2003) que estudaram apenas o peso à desmama de bovinos Nelore nas diversas regiões brasileiras e por Fridrich et al. (2005) para P205 nas regiões $\mathrm{S}$ e $\mathrm{SE}$ em bovinos Tabapuã. $\mathrm{O}$ valor da $h^{2}$ foi menor nas regiões S para P205 e S e $\mathrm{NE}$ para $\mathrm{P} 365$, em razão do aumento da $\sigma^{2}$, que foi proporcionalmente maior do que a $\sigma^{2}$ para essas regiões. Para P205, as regiões SE e CO apresentaram maiores estimativas de $\sigma_{a}^{2}$ e $h_{a}^{2}$, sugerindo que a seleção baseada no peso à desmama seria mais efetiva nessas regiões.

Apesar da variação observada nas estimativas de parâmetros genéticos para P205 e P365, os valores encontram-se na faixa de variação retratada na literatura para bovinos Nelore.

As estimativas das (co)variâncias genéticas aditivas direta e materna, fenotípicas e residuais de P205 e P365, consideradas características distintas nas diversas regiões, são apresentadas nas Tab. 4, 5, 6 e 7, respectivamente.

Tabela 4. Estimativas de variâncias genéticas aditiva direta (a1-a2) e materna (m1-m2) (diagonal) e covariâncias (fora da diagonal), obtidas pela análise conjunta do peso aos 205 (P205) dias de idade, consideradas como características distintas nas diversas combinações das regiões Sul (S), Sudeste (SE), Centro-Oeste (CO), Norte (N) e Nordeste (NE)

\begin{tabular}{|c|c|c|c|c|c|c|c|c|c|}
\hline & \multicolumn{4}{|c|}{ S e SE } & & \multicolumn{4}{|c|}{$\mathrm{S}$ e $\mathrm{CO}$} \\
\hline & a1 & $\mathrm{a} 2$ & $\mathrm{~m} 1$ & $\mathrm{~m} 2$ & & a1 & $\mathrm{a} 2$ & $\mathrm{ml}$ & $\mathrm{m} 2$ \\
\hline a1 & 69,37 & & & & al & 53,02 & & & \\
\hline $\mathrm{a} 2$ & 63,37 & 78,54 & & & $\mathrm{a} 2$ & 52,31 & 126,52 & & \\
\hline $\mathrm{m} 1$ & $-69,86$ & $-58,71$ & 126,07 & & $\mathrm{~m} 1$ & $-81,32$ & $-100,89$ & 130,60 & \\
\hline \multirow[t]{3}{*}{$\mathrm{m} 2$} & $-28,94$ & $-32,94$ & 91,26 & 88,71 & $\mathrm{~m} 2$ & 60,54 & $-60,06$ & 95,87 & 117,18 \\
\hline & \multicolumn{4}{|c|}{$\mathrm{S}$ e N } & & \multicolumn{4}{|c|}{ S e NE } \\
\hline & a1 & $\mathrm{a} 2$ & $\mathrm{~m} 1$ & $\mathrm{~m} 2$ & & a1 & a2 & $\mathrm{m} 1$ & $\mathrm{~m} 2$ \\
\hline a1 & 53,54 & & & & a1 & 37,21 & & & \\
\hline a2 & 60,65 & 122,95 & & & $\mathrm{a} 2$ & 43,73 & 83,28 & & \\
\hline $\mathrm{m} 1$ & $-81,32$ & $-100,89$ & 125,09 & & $\mathrm{~m} 1$ & $-65,12$ & $-70,68$ & 115,11 & \\
\hline \multirow[t]{3}{*}{$\mathrm{m} 2$} & $-58,01$ & $-90,53$ & 95,87 & 159,20 & $\mathrm{~m} 2$ & $-57,61$ & $-84,44$ & 97,74 & 97,97 \\
\hline & \multicolumn{4}{|c|}{$\mathrm{SE} \mathrm{e} \mathrm{CO}$} & & \multicolumn{4}{|c|}{ SE e N } \\
\hline & $\overline{\mathrm{a} 1}$ & $\mathrm{a} 2$ & $\mathrm{~m} 1$ & $\mathrm{~m} 2$ & & $\overline{\mathrm{a} 1}$ & $\mathrm{a} 2$ & $\mathrm{~m} 1$ & $\mathrm{~m} 2$ \\
\hline$\overline{\mathrm{a} 1}$ & 89,46 & & & & a1 & 80,48 & & & \\
\hline a2 & 98,35 & 128,75 & & & $\mathrm{a} 2$ & 76,46 & 80,28 & & \\
\hline $\mathrm{m} 1$ & $-34,35$ & $-59,58$ & 87,79 & & $\mathrm{~m} 1$ & $-25,97$ & $-8,67$ & 79,78 & \\
\hline \multirow[t]{3}{*}{$\mathrm{m} 2$} & $-49,48$ & $-69,69$ & 102,71 & 127,21 & $\mathrm{~m} 2$ & $-42,28$ & $-47,31$ & 59,27 & 125,69 \\
\hline & \multicolumn{4}{|c|}{ SE e NE } & & \multicolumn{4}{|c|}{$\mathrm{CO}$ e $\mathrm{N}$} \\
\hline & $\mathrm{a} 1$ & a2 & $\mathrm{m} 1$ & $\mathrm{~m} 2$ & & $\mathrm{a} 1$ & $\mathrm{a} 2$ & $\mathrm{ml}$ & $\mathrm{m} 2$ \\
\hline$\overline{a 1}$ & 77,36 & & & & a1 & 130,19 & & & \\
\hline a2 & 79,94 & 119,41 & & & $\mathrm{a} 2$ & 95,10 & 89,20 & & \\
\hline $\mathrm{m} 1$ & $-23,85$ & $-33,80$ & 79,79 & & $\mathrm{~m} 1$ & $-65,84$ & $-13,45$ & 121,23 & \\
\hline \multirow[t]{3}{*}{$\mathrm{m} 2$} & $-60,75$ & $-94,47$ & 71,90 & 104,31 & $\mathrm{~m} 2$ & $-106,82$ & $-50,52$ & 102,55 & 126,01 \\
\hline & \multicolumn{4}{|c|}{$\mathrm{CO}$ e $\mathrm{NE}$} & & \multicolumn{4}{|c|}{$\mathrm{Ne} \mathrm{NE}$} \\
\hline & $\overline{\mathrm{a} 1}$ & $\mathrm{a} 2$ & $\mathrm{~m} 1$ & $\mathrm{~m} 2$ & & $\overline{\mathrm{a} 1}$ & $\mathrm{a} 2$ & $\mathrm{ml}$ & $\mathrm{m} 2$ \\
\hline$\overline{a 1}$ & 113,52 & & & & a1 & 95,64 & & & \\
\hline $\mathrm{a} 2$ & 40,09 & 126,73 & & & $\mathrm{a} 2$ & 72,96 & 141,71 & & \\
\hline $\mathrm{m} 1$ & $-30,69$ & $-96,87$ & 94,97 & & $\mathrm{~m} 1$ & $-54,20$ & $-127,26$ & 129,36 & \\
\hline $\mathrm{m} 2$ & 26,54 & $-107,45$ & 96,48 & 137,32 & $\mathrm{~m} 2$ & $-93,05$ & $-127,56$ & 103,87 & 129,94 \\
\hline
\end{tabular}


Tabela 5. Estimativas de variâncias fenotípica (p1-p2) e residual (e1-e2) (diagonal) e covariâncias (fora da diagonal), obtidas pela análise conjunta do peso aos 205 (P205) dias de idade, consideradas como características distintas nas diversas combinações das regiões Sul (S), Sudeste (SE), Centro-Oeste (CO), Norte $(\mathrm{N})$ e Nordeste (NE)

\begin{tabular}{|c|c|c|c|c|c|c|c|c|c|}
\hline & \multicolumn{4}{|c|}{ S e SE } & & \multicolumn{4}{|c|}{$\mathrm{S}$ e $\mathrm{CO}$} \\
\hline & $\overline{\mathrm{p} 1}$ & $\mathrm{p} 2$ & e1 & e2 & & $\overline{\mathrm{p} 1}$ & $\mathrm{p} 2$ & el & e2 \\
\hline $\bar{p} 1$ & 400,70 & & & & p1 & 397,76 & & & \\
\hline p2 & $-268,07$ & 332,31 & & & p2 & $-204,03$ & 437,87 & & \\
\hline e1 & - & - & 275,13 & & e1 & - & - & 295,46 & \\
\hline \multirow[t]{3}{*}{$\mathrm{e} 2$} & - & - & $-268,07$ & 332,31 & e2 & - & - & $-271,50$ & 254,24 \\
\hline & \multicolumn{4}{|c|}{ S e N } & & \multicolumn{4}{|c|}{ S e NE } \\
\hline & $\overline{\mathrm{p} 1}$ & p2 & e1 & $\mathrm{e} 2$ & & $\overline{\mathrm{p} 1}$ & $\mathrm{p} 2$ & el & e2 \\
\hline$\overline{\mathrm{p} 1}$ & 410,48 & & & & p1 & 392,10 & & & \\
\hline p2 & $-181,51$ & 405,13 & & & $\mathrm{p} 2$ & $-202,37$ & 362,06 & & \\
\hline e1 & - & - & 131,17 & & e1 & - & - & 304,89 & \\
\hline \multirow[t]{3}{*}{$\mathrm{e} 2$} & - & - & $-258,57$ & 213,50 & $\mathrm{e} 2$ & - & - & $-279,69$ & 265,24 \\
\hline & \multicolumn{4}{|c|}{$\mathrm{SE}$ e $\mathrm{CO}$} & & \multicolumn{4}{|c|}{ SE e N } \\
\hline & $\mathrm{p} 1$ & $\mathrm{p} 2$ & el & e2 & & $\overline{\mathrm{p} 1}$ & $\mathrm{p} 2$ & el & e2 \\
\hline$\overline{\mathrm{p} 1}$ & 469,73 & & & & $\mathrm{p} 1$ & 468,37 & & & \\
\hline p2 & $-127,03$ & 439,12 & & & p2 & $-171,14$ & 395,69 & & \\
\hline el & - & - & 326,83 & & el & - & - & 334,08 & \\
\hline \multirow[t]{3}{*}{$\mathrm{e} 2$} & - & - & $-273,55$ & 252,85 & $\mathrm{e} 2$ & - & - & $-281,40$ & 237,03 \\
\hline & \multicolumn{4}{|c|}{ SE e NE } & & \multicolumn{4}{|c|}{$\mathrm{CO}$ e $\mathrm{N}$} \\
\hline & $\overline{\mathrm{p} 1}$ & p2 & e1 & e2 & & $\overline{\mathrm{p} 1}$ & $\mathrm{p} 2$ & e1 & e2 \\
\hline$\overline{\mathrm{p} 1}$ & 466,58 & & & & $\mathrm{p} 1$ & 438,52 & & & \\
\hline p2 & 384,92 & 370,19 & & & $\mathrm{p} 2$ & 248,14 & 396,59 & & \\
\hline e1 & - & - & 333,28 & & el & - & - & 252,93 & \\
\hline \multirow[t]{3}{*}{ e2 } & - & - & 280,36 & 240,95 & $\mathrm{e} 2$ & - & - & 110,63 & 231,89 \\
\hline & \multicolumn{4}{|c|}{$\mathrm{CO}$ e $\mathrm{NE}$} & & \multicolumn{4}{|c|}{$\mathrm{Ne} \mathrm{NE}$} \\
\hline & $\overline{\mathrm{p} 1}$ & p2 & el & $\mathrm{e} 2$ & & $\overline{\mathrm{p} 1}$ & p2 & el & e2 \\
\hline$\overline{\mathrm{p} 1}$ & 437,61 & & & & p1 & 397,91 & & & \\
\hline p2 & $-121,96$ & 381,67 & & & $\mathrm{p} 2$ & $-168,01$ & 374,38 & & \\
\hline e1 & - & - & 259,80 & & e1 & - & - & 227,11 & \\
\hline e2 & - & - & $-223,37$ & 225,07 & $\mathrm{e} 2$ & - & - & $-228,69$ & 230,28 \\
\hline
\end{tabular}

Tabela 6. Estimativas de variâncias genéticas aditiva direta (a1-a2) e materna (m1-m2) (diagonal) e covariâncias (fora da diagonal), obtidas pela análise conjunta do peso aos 365 (P365) dias de idade, consideradas como características distintas nas diversas combinações das regiões Sul (S), Sudeste (SE), Centro-Oeste (CO), Norte $(\mathrm{N})$ e Nordeste (NE)

\begin{tabular}{|c|c|c|c|c|c|c|c|c|c|}
\hline & \multicolumn{4}{|c|}{ S e SE } & & \multicolumn{4}{|c|}{$\mathrm{S}$ e $\mathrm{CO}$} \\
\hline & $\mathrm{a} 1$ & $\mathrm{a} 2$ & $\mathrm{ml}$ & $\mathrm{m} 2$ & & $\mathrm{a} 1$ & $\mathrm{a} 2$ & $\mathrm{~m} 1$ & M2 \\
\hline a1 & 38,53 & & & & a1 & 59,27 & & & \\
\hline $\mathrm{a} 2$ & 51,69 & 89,80 & & & $\mathrm{a} 2$ & 36,95 & 192,33 & & \\
\hline $\mathrm{m} 1$ & $-5,63$ & $-56,45$ & 1229,01 & & $\mathrm{~m} 1$ & $-64,29$ & 48,61 & 152,90 & \\
\hline \multirow[t]{3}{*}{$\mathrm{m} 2$} & 12,81 & $-25,15$ & 105,32 & 94,08 & $\mathrm{~m} 2$ & 51,71 & $-70,84$ & $-90,76$ & 118,05 \\
\hline & \multicolumn{4}{|c|}{$\mathrm{S}$ e $\mathrm{N}$} & & \multicolumn{4}{|c|}{ S e NE } \\
\hline & $\overline{\mathrm{a} 1}$ & a2 & $\mathrm{m} 1$ & $\mathrm{~m} 2$ & & $\overline{\mathrm{a} 1}$ & $\mathrm{a} 2$ & $\mathrm{~m} 1$ & $\mathrm{~m} 2$ \\
\hline$\overline{\text { a1 }}$ & 118,21 & & & & a1 & 89,19 & & & \\
\hline a2 & 55,39 & 145,65 & & & $\mathrm{a} 2$ & 55,69 & 46,89 & & \\
\hline $\mathrm{m} 1$ & $-80,79$ & 12,47 & 149,11 & & $\mathrm{~m} 1$ & $-62,39$ & $-4,27$ & 143,04 & \\
\hline \multirow[t]{3}{*}{$\mathrm{m} 2$} & $-90,41$ & $-14,13$ & 69,53 & 76,03 & $\mathrm{~m} 2$ & $-90,49$ & $-58,27$ & 58,23 & 92,06 \\
\hline & \multicolumn{4}{|c|}{ SE e CO } & & \multicolumn{4}{|c|}{ SE e N } \\
\hline & a1 & a2 & $\mathrm{m} 1$ & $\mathrm{~m} 2$ & & $\overline{\mathrm{a} 1}$ & $\mathrm{a} 2$ & $\mathrm{~m} 1$ & $\mathrm{~m} 2$ \\
\hline a1 & 104,68 & & & & a1 & 94,36 & & & \\
\hline $\mathrm{a} 2$ & 111,64 & 181,92 & & & $\mathrm{a} 2$ & 100,41 & 154,73 & & \\
\hline $\mathrm{m} 1$ & 4,59 & $-41,82$ & 61,88 & & $\mathrm{~m} 1$ & $-2,23$ & $-43,63$ & 72,16 & \\
\hline \multirow[t]{3}{*}{$\mathrm{m} 2$} & 1,95 & $-65,47$ & 76,75 & 117,05 & $\mathrm{~m} 2$ & 19,04 & $-35,66$ & 80,98 & 99,39 \\
\hline & \multicolumn{4}{|c|}{ SE e NE } & & \multicolumn{4}{|c|}{$\mathrm{CO}$ e $\mathrm{N}$} \\
\hline & $\overline{\mathrm{a} 1}$ & $\mathrm{a} 2$ & $\mathrm{ml}$ & $\mathrm{m} 2$ & & $\overline{\mathrm{a} 1}$ & $\mathrm{a} 2$ & $\mathrm{ml}$ & $\mathrm{m} 2$ \\
\hline a1 & 83,12 & & & & a1 & 114,44 & & & \\
\hline a2 & 84,34 & 85,34 & & & $\mathrm{a} 2$ & 84,34 & 94,36 & & \\
\hline $\mathrm{m} 1$ & 8,89 & 10,92 & 64,54 & & $\mathrm{~m} 1$ & $-22,73$ & 10,92 & 84,82 & \\
\hline \multirow[t]{3}{*}{$\mathrm{m} 2$} & $-35,60$ & $-33,97$ & 68,66 & 97,85 & $\mathrm{~m} 2$ & $-38,70$ & $-33,96$ & 68,66 & 90,26 \\
\hline & \multicolumn{4}{|c|}{$\mathrm{CO}$ e $\mathrm{NE}$} & & \multicolumn{4}{|c|}{$\mathrm{Ne} \mathrm{NE}$} \\
\hline & $\overline{\mathrm{a} 1}$ & $\mathrm{a} 2$ & $\mathrm{~m} 1$ & $\mathrm{~m} 2$ & & $\overline{\mathrm{a} 1}$ & $\mathrm{a} 2$ & $\mathrm{~m} 1$ & $\mathrm{~m} 2$ \\
\hline$\overline{a 1}$ & 187,20 & & & & al & 148,06 & & & \\
\hline a2 & 78,34 & 45,88 & & & $\mathrm{a} 2$ & 86,55 & 51,26 & & \\
\hline $\mathrm{m} 1$ & $-59,01$ & 9,09 & 107,58 & & $\mathrm{~m} 1$ & 7,61 & 10,22 & 51,88 & \\
\hline $\mathrm{m} 2$ & $-112,36$ & $-42,88$ & 43,99 & 71,20 & $\mathrm{~m} 2$ & $-111,94$ & $-65,02$ & 1,32 & 92,34 \\
\hline
\end{tabular}


Tabela 7. Estimativas de variâncias fenotípica (p1-p2) e residual (e1-e2) (diagonal) e covariâncias (fora da diagonal), obtidas pela análise conjunta do peso aos 365 (P365) dias de idade, consideradas como características distintas nas diversas combinações das regiões Sul (S), Sudeste (SE), Centro-Oeste (CO), Norte $(\mathrm{N})$ e Nordeste (NE)

\begin{tabular}{|c|c|c|c|c|c|c|c|c|c|}
\hline & \multicolumn{4}{|c|}{ S e SE } & & \multicolumn{4}{|c|}{$\mathrm{S}$ e $\mathrm{CO}$} \\
\hline & $\overline{\mathrm{p} 1}$ & p2 & el & e2 & & $\overline{\mathrm{p} 1}$ & p2 & e1 & e2 \\
\hline$\overline{\mathrm{p} 1}$ & 553,94 & & & & p1 & 539,24 & & & \\
\hline p2 & 594,87 & 707,34 & & & p2 & 398,01 & 653,05 & & \\
\hline e1 & - & - & 392,04 & & e1 & - & - & 391,37 & \\
\hline \multirow[t]{3}{*}{$111 \mathrm{e} 2$} & - & - & 459,67 & 539,60 & $\mathrm{e} 2$ & - & - & 401,66 & 413,51 \\
\hline & \multicolumn{4}{|c|}{ S e N } & & \multicolumn{4}{|c|}{ S e NE } \\
\hline & $\overline{\mathrm{p} 1}$ & p2 & e1 & e2 & & $\overline{\mathrm{p} 1}$ & $\mathrm{p} 2$ & $\mathrm{e} 1$ & e2 \\
\hline$\overline{\mathrm{p} 1}$ & 551,43 & & & & $\mathrm{p} 1$ & 546,92 & & & \\
\hline p2 & 266,46 & 681,28 & & & p2 & 483,58 & 571,72 & & \\
\hline e1 & - & - & 364,91 & & $\mathrm{e} 1$ & - & - & 377,07 & \\
\hline \multirow[t]{3}{*}{$\mathrm{e} 2$} & - & - & 180,50 & 473,74 & $\mathrm{e} 2$ & - & - & 417,04 & 491,05 \\
\hline & \multicolumn{4}{|c|}{$\mathrm{SE}$ e $\mathrm{CO}$} & & \multicolumn{4}{|c|}{ SE e N } \\
\hline & $\mathrm{p} 1$ & p2 & e1 & e2 & & $\overline{\mathrm{p} 1}$ & $\mathrm{p} 2$ & $\mathrm{e} 1$ & $\mathrm{e} 2$ \\
\hline$\overline{\mathrm{p} 1}$ & 708,62 & & & & $\mathrm{p} 1$ & 706,50 & & & \\
\hline p2 & 581,49 & 651,62 & & & p2 & 671,26 & 683,55 & & \\
\hline e1 & - & - & 537,47 & & $\mathrm{e} 1$ & - & - & 542,22 & \\
\hline \multirow[t]{3}{*}{ e2 } & - & - & 413,03 & 418,12 & e2 & - & - & 502,17 & 465,08 \\
\hline & \multicolumn{4}{|c|}{ SE e NE } & & \multicolumn{4}{|c|}{$\mathrm{CO}$ e $\mathrm{N}$} \\
\hline & $\overline{\mathrm{p} 1}$ & p2 & e1 & $\mathrm{e} 2$ & & $\overline{\mathrm{p} 1}$ & $\mathrm{p} 2$ & e1 & $\mathrm{e} 2$ \\
\hline$\overline{\mathrm{p} 1}$ & 709,63 & & & & $\mathrm{p} 1$ & 647,56 & & & \\
\hline p2 & 650,85 & 620,15 & & & p2 & 649,30 & 703,25 & & \\
\hline e1 & - & - & 553,08 & & e1 & - & - & 471,03 & \\
\hline \multirow[t]{3}{*}{ e2 } & - & - & 510,19 & 470,62 & e2 & - & - & 510,19 & 552,60 \\
\hline & \multicolumn{4}{|c|}{$\mathrm{CO}$ e $\mathrm{NE}$} & & \multicolumn{4}{|c|}{$\mathrm{Ne} \mathrm{NE}$} \\
\hline & p1 & p2 & e1 & e2 & & p1 & p2 & e1 & e2 \\
\hline p1 & 652,00 & & & & $\mathrm{p} 1$ & 682,07 & & & \\
\hline p2 & 358,12 & 568,83 & & & p2 & 521,21 & 572,71 & & \\
\hline e1 & - & - & 416,22 & & e1 & - & - & 474,52 & \\
\hline $\mathrm{e} 2$ & - & - & 287,41 & 494,63 & $\mathrm{e} 2$ & - & - & 484,21 & 494,12 \\
\hline
\end{tabular}

Em geral, ocorreu ampla variação nas estimativas de variâncias e covariâncias genéticas direta, materna e fenotípica, tanto para P205 quanto para P365 em análises conjuntas, considerando-se cada uma das características como característica distinta nas combinações de pares de regiões estudadas, o que pode ser atribuído à expressiva variação no número de informações disponíveis em cada região, principalmente quando são cotejados os números de dados disponíveis nas regiões $\mathrm{S}, \mathrm{N}$ e $\mathrm{NE}$ com os disponíveis nas regiões $\mathrm{SE}$ e $\mathrm{CO}$ que representam $87,17 \%$ de todos os dados.

As estimativas de herdabilidade e correlações genéticas dos pesos aos 205 dias de idade (P205) e aos trezentos e sessenta e cinco dias de idade (P365), cada um deles considerado característica distinta nas diversas regiões, obtidas em análises bicaracterísticas, são apresentadas nas Tab. 8 e 9, respectivamente.

As correlações genéticas encontradas para P205 e P365, ambos considerados características distintas nas diversas regiões, indicam que, na desmama (P205), o efeito da IGA não foi observado somente nas combinações que envolveram a região SE com as demais regiões e em $\mathrm{CO} / \mathrm{N}$. Para as demais regiões o efeito da IGA foi importante.
Considerando a característica P365, o efeito da interação foi evidenciado somente nas combinações $\mathrm{S} / \mathrm{CO}$ e $\mathrm{S} / \mathrm{N}$. Nas demais regiões não houve efeito da IGA. As estimativas de correlações genéticas para a maioria das combinações de regiões foram altas, sugerindo que grande parte dos genes de ação aditiva que influencia uma característica também influencia a outra. De acordo com Robertson (1959), quando o valor da correlação genética for menor que 0,8 , a interação genótipo $\mathrm{x}$ ambiente passa a assumir papel importante na expressão da característica.

Fridrich et al. (2005), ao avaliarem pesos de bovinos Tabapuã nas diversas regiões brasileiras, também encontraram interação genótipo ambiente para P205 nas combinações que envolviam as regiões $\mathrm{S}$ e $\mathrm{NE}$ e $\mathrm{SE}$ e $\mathrm{NE}$ e, para P365, em todas as combinações que envolviam a região $\mathrm{NE}$

As altas correlações observadas nas Tab. $8(0,95)$ para SE/N e Tab. 9 para SE/NE $(1,00)$ e N/NE $(0,99)$, próximas ou iguais a um, mesmo considerando número diferente de informações nas regiões, indicam que os mesmos grupos de genes são responsáveis pela expressão das duas características estudadas. 
Tabela 8. Estimativas de herdabilidade direta para as características $1\left(\mathrm{~h}_{\mathrm{a}}^{2} 1\right)$ e $2\left(\mathrm{~h}_{\mathrm{a}}^{2} 2\right)$, herdabilidade materna para as características $1\left(\mathrm{~h}_{\mathrm{m}}^{2} 1\right)$ e $2\left(\mathrm{~h}_{\mathrm{m}}^{2} 2\right)$, e correlações genéticas dos efeitos aditivos diretos $\left(\mathrm{r}_{\mathrm{g}}\right)$ dos pesos aos 205 (P205) dias de idade, considerados como características distintas nas diversas combinações das regiões Sul (S), Sudeste (SE), Centro-Oeste (CO), Norte (N) e Nordeste (NE)

\begin{tabular}{lllllllllll}
\hline Característica & $\mathrm{S} / \mathrm{SE}$ & $\mathrm{S} / \mathrm{CO}$ & $\mathrm{S} / \mathrm{N}$ & $\mathrm{S} / \mathrm{NE}$ & $\mathrm{SE} / \mathrm{CO}$ & $\mathrm{SE} / \mathrm{N}$ & $\mathrm{SE} / \mathrm{NE}$ & $\mathrm{CO} / \mathrm{N}$ & $\mathrm{CO} / \mathrm{NE}$ & $\mathrm{N} / \mathrm{NE}$ \\
\hline $\mathrm{h}^{2}{ }_{\mathrm{a}} 1$ & 0,17 & 0,13 & 0,13 & 0,09 & 0,19 & 0,17 & 0,17 & 0,30 & 0,26 & 0,24 \\
$\mathrm{~h}^{2}{ }_{\mathrm{a}}{ }^{2}$ & 0,17 & 0,29 & 0,30 & 0,23 & 0,29 & 0,20 & 0,32 & 0,22 & 0,33 & 0,38 \\
$\mathrm{~h}^{2}{ }_{\mathrm{m}} 1$ & 0,31 & 0,33 & 0,30 & 0,29 & 0,19 & 0,17 & 0,17 & 0,28 & 0,22 & 0,33 \\
$\mathrm{~h}^{2}{ }_{\mathrm{m}}{ }^{2}$ & 0,19 & 0,27 & 0,39 & 0,27 & 0,29 & 0,32 & 0,28 & 0,32 & 0,36 & 0,35 \\
$\mathrm{r}_{\mathrm{g}}$ & 0,86 & 0,64 & 0,75 & 0,79 & 0,92 & 0,95 & 0,83 & 0,88 & 0,33 & 0,63 \\
\hline
\end{tabular}

Tabela 9. Estimativas de herdabilidade direta para as características $1\left(\mathrm{~h}_{\mathrm{a}}^{2} 1\right)$ e $2\left(\mathrm{~h}_{\mathrm{a}}^{2} 2\right)$, herdabilidade materna para as características $1\left(\mathrm{~h}_{\mathrm{m}}^{2} 1\right)$ e $2\left(\mathrm{~h}_{\mathrm{m}}^{2} 2\right)$, e correlações genéticas dos efeitos aditivos diretos $\left(\mathrm{r}_{\mathrm{g}}\right)$ dos pesos aos 365 (P365) dias de idade considerados como características distintas nas diversas combinações das regiões Sul (S), Sudeste (SE), Centro-Oeste (CO), Norte (N) e Nordeste (NE)

\begin{tabular}{lllllllllll} 
Característica & $\mathrm{S} / \mathrm{SE}$ & $\mathrm{S} / \mathrm{CO}$ & $\mathrm{S} / \mathrm{N}$ & $\mathrm{S} / \mathrm{NE}$ & $\mathrm{SE} / \mathrm{CO}$ & $\mathrm{SE} / \mathrm{N}$ & $\mathrm{SE} / \mathrm{NE}$ & $\mathrm{CO} / \mathrm{N}$ & $\mathrm{CO} / \mathrm{NE}$ & $\mathrm{N} / \mathrm{NE}$ \\
\hline $\mathrm{h}^{2}{ }_{\mathrm{a}} 1$ & 0,07 & 0,11 & 0,21 & 0,16 & 0,15 & 0,13 & 0,12 & 0,18 & 0,29 & 0,22 \\
$\mathrm{~h}^{2}{ }_{\mathrm{a}} 2$ & 0,14 & 0,29 & 0,21 & 0,08 & 0,28 & 0,23 & 0,14 & 0,13 & 0,08 & 0,09 \\
$\mathrm{~h}^{2}{ }_{\mathrm{m}} 1$ & 0,23 & 0,28 & 0,27 & 0,26 & 0,09 & 0,10 & 0,09 & 0,13 & 0,17 & 0,08 \\
$\mathrm{~h}^{2}{ }_{\mathrm{m}} 2$ & 0,13 & 0,18 & 0,11 & 0,16 & 0,18 & 0,15 & 0,16 & 0,13 & 0,13 & 0,16 \\
$\mathrm{r}_{\mathrm{g}}$ & 0,84 & 0,35 & 0,42 & 0,86 & 0,81 & 0,83 & 1,00 & 0,81 & 0,85 & 0,99 \\
\hline
\end{tabular}

De maneira geral, as estimativas das herdabilidades (moderadas) dos pesos P205 e P365 foram semelhantes e indicam que a raça Nelore apresenta variabilidade genética aditiva suficiente para que ocorra resposta à seleção baseada nestes pesos. Ferraz Filho et al. (2002), entretanto, observaram diminuição da magnitude das herdabilidades com o avanço da idade dos animais, em decorrência do menor número de observações com o avanço da idade, o que redunda na obtenção de estimativas de herdabilidades menos precisas e acuradas.

Os altos valores das herdabilidades maternas para P205 sugerem que o efeito materno deve ser incluído nos modelos de avaliação genética em razão da sua expressiva participação nesta fase.

As correlações de posto entre os valores genéticos dos 62 melhores reprodutores com mais de 150 filhos em pelo menos três regiões, obtidas em análise bicaracterística, do P205 e P365 dias de idade, são apresentadas na Tab.10.

Os resultados das correlações de posto, ao se considerar o P205 ou o P365, cada uma como característica distinta nas diversas regiões, indicam que os reprodutores, em análise envolvendo pares de região, foram ordenados (geneticamente) de forma semelhante quando se consideraram as combinações das regiões S/SE, $\mathrm{S} / \mathrm{NE}, \mathrm{SE} / \mathrm{N}, \mathrm{SE} / \mathrm{NE}$ e CO/N. Porém, quando se consideraram as combinações $\mathrm{S} / \mathrm{CO}$ e $\mathrm{S} / \mathrm{N}$, o mesmo não foi verificado. Os resultados indicaram baixas correlações de posto 0,71 (P205) e 0,73 (P365) para S/CO e 0,78 (P205) e 0,78 (P365) para S/N. Quando se considerou P205 para as combinações S/CO, S/N, SE/CO, $\mathrm{SE} / \mathrm{NE}, \mathrm{CO} / \mathrm{NE}$ e N/NE, os valores das correlações variaram de 0,71 a 0,79 , o que mostra que a classificação genética de um reprodutor em uma região seria diferente da realizada em outra região. Quando se considerou P365, somente as combinações que envolveram as regiões $\mathrm{S} / \mathrm{CO}, \mathrm{S} / \mathrm{N}$ e $\mathrm{SE} / \mathrm{CO}$ apresentaram correlações de posto com valores de $0,73,0,78$ e 0,76 que são menores que 0,80 . Estes resultados reforçam a idéia de que os reprodutores deveriam ser testados por região, principalmente quando se consideram regiões muito distintas. As grandes diferenças climáticas e nutricionais em que os animais são submetidos em cada região podem ser responsáveis pelos diferentes desempenhos.

Fridrich et al. (2005) encontraram correlação de posto para P205 em bovinos Tabapuã iguais a 0,81 quando consideraram a combinação das regiões S e NE, para P365 dias de idade as correlações de posto foram iguais a $-0,51$ para as regiões S e NE, 0,76 para $\mathrm{SE}$ e NE e 0,53 para as regiões $\mathrm{CO}$ e NE. Esses resultados indicam que os reprodutores com melhores resultados nas regiões $\mathrm{S}, \mathrm{SE}$ e $\mathrm{CO}$ não apresentam o mesmo desempenho da região NE. 
Tabela 10. Correlação de posto entre os valores genéticos dos touros, obtida a partir de análises bicaracterísticas entre o par de combinação das regiões Sul (S), Sudeste (SE), Centro-Oeste (CO), Norte (N) e Nordeste(NE)

\begin{tabular}{lcc}
\multicolumn{1}{c}{$\begin{array}{c}\text { Combinação de } \\
\text { regiões }\end{array}$} & \multicolumn{2}{c}{ Correlação de posto } \\
\cline { 2 - 3 } $\mathrm{S} / \mathrm{SE}$ & $\mathrm{P} 205$ & $\mathrm{P} 365$ \\
$\mathrm{~S} / \mathrm{CO}$ & 0,89 & 0,80 \\
$\mathrm{~S} / \mathrm{N}$ & 0,71 & 0,73 \\
$\mathrm{~S} / \mathrm{NE}$ & 0,78 & 0,78 \\
$\mathrm{SE} / \mathrm{CO}$ & 0,82 & 0,84 \\
$\mathrm{SE} / \mathrm{N}$ & 0,76 & 0,76 \\
$\mathrm{SE} / \mathrm{NE}$ & 0,81 & 0,89 \\
$\mathrm{CO} / \mathrm{N}$ & 0,79 & 0,96 \\
$\mathrm{CO} / \mathrm{NE}$ & 0,73 & 0,87 \\
$\mathrm{~N} / \mathrm{NE}$ & 0,79 & 0,86 \\
\hline
\end{tabular}

Buchanan e Nielsen (1979) e Bertrand et al. (1985) observaram efeitos das interações touro $\mathrm{x}$ região, touro $\mathrm{x}$ rebanho/região e touro $\mathrm{x} \mathrm{GC} /$ região sobre a ordem de classificação dos animais entre as regiões, rebanhos e GC dentro de região, quando foram analisados os pesos ao nascimento e ao desmame dos animais, respectivamente. Matos et al. (1996) observaram, em bovinos Hereford, que a interação reprodutor $\mathrm{x}$ região dentro de país (Uruguai) foi grande o suficiente para causar mudanças na ordem de classificação dos reprodutores entre regiões.

A existência da IGA encontrada nas combinações que envolveram as regiões $\mathrm{S}, \mathrm{SE}, \mathrm{CO}, \mathrm{N}$ e $\mathrm{NE}$ indicam que a utilização de modelos de avaliação que consideram a IGA seria de grande valia para maior qualidade das predições de valores genéticos em diferentes regiões.

\section{CONCLUSÕES}

Não se observa interação genótipo $\mathrm{x}$ ambiente para pesos aos 205 e 365 dias de idade quando se consideram somente as regiões Sudeste e CentroOeste, que representam $87,17 \%$ dos dados. Os resultados obtidos sinalizam a necessidade de avaliação genética regional quando se consideram observações de regiões $\mathrm{S}, \mathrm{N}$ ou NE.

\section{REFERÊNCIAS BIBLIOGRÁFICAS}

ALENCAR, M.M.; MASCIOLI, A.S.; FREITAS, A.R. Evidências de interação genótipo $\mathrm{x}$ ambiente sobre características de crescimento de bovinos de corte. Rev. Bras. Zootec., v.34, p.489-495, 2005.

BERTRAND, J.K.; BERGER, P.J.; WILHAM, R.L. Sire x environment interactions in beef cattle weaning weight field data. J. Anim. Sci., v.60, p.1397-1402, 1985.
BOLDMAN, K.G.; KRIESE, L.A.; VAN VLECK, L.D. et al. A manual for use of MTDFREML : a set of programs to obtain estimates of variance and covariance. Lincoln : Agricultural Research Service, 1995. 120p.

BUCHANAN, D.S.; NIELSEN, M.K. Sire by environment interactions in beef cattle field data. J. Anim. Sci., v.48, p.307-312, 1979.

FALCONER, D.S. The problem of environment and selection. Am. Nat., v.86, p.293-298, 1952.

FERRAZ FILHO, P.B.; RAMOS, A.A.; SILVA, L.O.C. et al. Tendência genética dos efeitos direto e materno sobre os pesos à desmama e pós-desmama de bovinos da raça Tabapuã. Rev. Bras. Zootec., v.31, p.635-640, 2002.

FERREIRA, V.C.P.; PENNA, V.M.; BERGMANN, J.A.G. ET AL. Interação genótipo-ambiente em algumas características produtivas de gado de corte no brasil. Arq. Bras. Med. Vet. Zootec., v.53, p.385-392, 2001.

FRIDRICH, A.B.; SILVA, M.A.; FRIDRICH, D. et al. Interação genótipo $\mathrm{x}$ ambiente e estimativas de parâmetros genéticos de características ponderais de bovinos Tabapuã. Arq. Bras. Med. Vet. Zootec., v.57, p.663-672, 2005.

HAMMOND, J. Animal breeding in relation to nutrition and environment conditions. J. Anim. Sci., v.22, p.195-213, 1947.

MARQUES, L.F.A.; PEREIRA, J.C.C.; OLIVEIRA, H.N. et al. Análise de características de crescimento da raça Simental. Arq. Bras. Med. Vet. Zootec., v.52, p.527-533, 2000 .

MATOS, S.D.; BERTRAND, J.K.; HERRING, W.O. et al. Sire and maternal grandsire by environment interaction for weaning weight in a Hereford beef cattle population in Uruguay. J. Anim. Sci., v.74, suppl.1, p.106, 1996. (Abstract).

PEREIRA, E.; ELER, J.P.; FERRAZ, J.B.S. Análise genética de algumas características reprodutivas e suas relações com o desempenho ponderal na raça Nelore. Arq. Bras. Med. Vet. Zootec., v.53, p.720-727, 2001.

RIBEIRO, M.N.; PIMENTA FILHO, E.C.; MARTINS, G.A. et al. Herdabilidade para efeitos diretos e maternos de características de crescimento de bovinos Nelore no estado da Paraíba. Rev. Bras. Zootec., v.30, p.1224- 1227, 2001.

RIBEIRO, S. Estudo da interação genótipos $\mathrm{x}$ ambiente em algumas características produtivas na raça Nelore. 2006. 72f. Tese (Doutorado) - Faculdade de Zootecnia e Engenharia de Alimentos, Universidade de São Paulo, Pirassununga, SP.

ROBERTSON, A. The sampling variance of the genetic correlation coefficient. Biometrics, v.15, p.469-485, 1959.

SOUZA, J.C.; GADINI, C.H.; SILVA, L.O.C. et al. Estimates of genetic parameters and evaluation os genotype $\mathrm{x}$ environment interaction for weaning weight in Nellore cattle. Arch. Latinoam. Prod. Anim., v.11, p.94-100, 2003.

STATISTICAL analysis system. Versão 6.12 para windows. Cary, NC: SAS Institute, 1997. 1290p.

VALENTE, B.D.; SILVA, M.A.; SILVA, L.O.C. et al. Estruturas de Covariância de peso em função da idade de animais Nelore das regiões Sudeste e Centro-Oeste do Brasil. Arq. Bras. Med. Vet. Zootec., v.60, p.389-400, 2008. 